ДИНАМИКА КЛИНИЧЕСКИХ ПРОЯВЛЕНИЙ И ЭКГ-ИЗМЕНЕНИЙ ТРОМБОЭМБОЛИИ ЛЕГОЧНОЙ АРТЕРИИ У БОЛЬНЫХ БЕЗ АРТЕРИАЛЬНОЙ ГИПОТЕНЗИИ В ОСТРЕЙШЕМ ПЕРИОДЕ

\author{
А. А. Ермолаев ${ }^{1,2}$, Н. Ф. Плавунов ${ }^{3}$, Е. А. Спиридонова ${ }^{1,4,5}$, Л. Л. Стажадзе ${ }^{1}$ \\ ${ }^{1}$ ФГУ Учебно-научный медицинский центр Управления делами Президента Российской Федерации, \\ кафедра скорой медицинской помощи и интенсивной терапии \\ ${ }^{2}$ Государственное бюджетное учреждение Станция скорой \\ и неотложной медицинской помощи им. А. С. Пучкова Департамента здравоохранения г. Москвы \\ ${ }^{3}$ Департамент Здравоохранения города Москвы \\ ${ }^{4}$ Московский государственный медико-стоматологический университет, \\ кафедра анестезиологии-реаниматологии \\ 5 Федеральный научно-клинический Центр детской гематологии, \\ онкологии и иммунологии Минздравсоцразвития России
}

\title{
Changes in the Clinical and ECG Pattern of Pulmonary Artery Thromboembolism in Patients without Arterial Hypotension in the Acutest Period
}

\author{
A. A. Ermolayev ${ }^{1,2}$, N. F. Plavunov ${ }^{3}$, E. A. Spiridonova ${ }^{1,4,5}$ L. L. Stazhadze ${ }^{1}$ \\ ${ }^{1}$ Department of Emergency Medical Care and Intensive Therapy, Training and Research Center, \\ Department for Presidential Affairs of the Russian Federation; \\ 2A. S. Puchkov Station of Emergency and Urgent Medical Care, Moscow Department of Healthcare; \\ ${ }^{3}$ Moscow Department of Healthcare \\ ${ }^{4}$ Department of Anesthesiology and Reanimatology, Moscow State University of Medicine and Dentistry; \\ ${ }^{5}$ Federal Research and Clinical Center of Pediatric Hematology, Oncology, and Immunology, \\ Ministry of Health and Social Development of Russia
}

\begin{abstract}
Цель исследования - оценить динамику состояния функций дыхания и кровообращения в острейшем периоде у больных с ТЭЛА без артериальной гипотонии при первичном обращении. Материал и методы. Проведен анализ 28 случаев ТЭЛА без снижения артериального давления при первичном осмотре на догоспитальном этапе (САД не менее 100 мм рт. ст.) В ис-

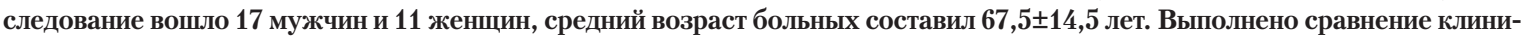
ческой и ЭКГ-картины при первичном осмотре и в динамике (в среднем через $87,0 \pm 29,7$ мин). Результаты. На фоне проведенной бригадами СМП терапии частота тахипноэ уменьшилась с 75 при первичном осмотре до $64 \%$ в динамике $(p=0,07)$. Средняя ЧДД уменьшилась с $24,0 \pm 5,7$ до $21,0 \pm 5,6$ в мин $(p=0,02)$. Проявления цианоза также уменьшились с 39 до $32 \%$ $(p=0,4)$. Средние ЧСС, САД и ДАД составили при первичном осмотре 100,0 $\pm 19,1$ в мин, $140,0 \pm 21,9$ мм рт. ст. и 80,0 11,5 мм рт. ст., соответственно; после проведенной на догоспитальном этапе терапии $-100,0 \pm 28,2$ в мин, $130,0 \pm 36,8$ мм рт. ст. и $80,0 \pm 21,9$ мм рт. ст., соответственно ( $p>0,5)$. Тахикардия наблюдалась у $60 \%$ больных, как при первичном осмотре, так и после терапии. В динамике у 1 больного (4\%) развилась гипотония. При анализе ЭКГ выявлено нарастание в динамике признаков перегрузки правых отделов сердца. Заключение. Летальный исход среди больных с ТЭЛА без артериальной гипотонии на догоспитальном этапе зафиксирован в 1 случае (4\%), общая летальность составила 18\%. Установлено прогрессивное ухудшение тяжести состояния $32 \%$ больных за счет нарастания явлений как дыхательной, так и сердечно-сосудистой недостаточности, которые поддаются коррекции на догоспитальном этапе не более чем в 32 и $39 \%$ случаев, соответственно. Ключевые слова: тромбоэмболия легочной артерии, догоспитальный этап, летальность.
\end{abstract}

Objective: to estimate changes in respiratory and circulatory functions in the acutest period in patients with pulmonary artery thromboembolism (PATE) without arterial hypotension during their first visit. Subjects and methods. The authors analyzed 28 cases of PATE without lowered blood pressure (BP) on primary examination in the prehospital stage (systolic BP (SBP) was less than $100 \mathrm{~mm} \mathrm{Hg}$ ). The study enrolled 17 men and 11 women; their mean age was $67.5 \pm 14.5$ years. The clinical and ECG pattern was compared on primary examination and over time (following an average of $87 \pm 29.7 \mathrm{~min}$ ). Results. With emergency team care, the rate of tachypnea decreased from $75 \%$ on primary examination to $64 \%$ over time $(p=0.07)$. Mean respiration rate $(\mathrm{RR})$ fell from $24.0 \pm 5.7$ to $21.0 \pm 5.6$ per $\min (p=0.02)$. The manifestations of cyanosis were also reduced from 39 to $32 \%$ $(p=0.4)$. On primary examination, mean RR, SBP, and diastolic BP were $100.0 \pm 19.1$ per min, $140.0 \pm 21.9$ and $80.0 \pm 11.5 \mathrm{~mm}$ $\mathrm{Hg}$, respectively; after prehospital therapy, these were $100.0 \pm 28.2$ per $\mathrm{min}, 130.0 \pm 36.8$ and $80.0 \pm 21.9 \mathrm{~mm} \mathrm{Hg}$, respectively $(p>0.5)$. Tachycardia was observed in $60 \%$ of the patients both on primary examination and following therapy. One (4\%) patient, over time, developed hypotension. ECG analysis revealed an increase in the signs of right cardiac cavity overload. Conclusion. A prehospital fatal outcome was recorded in 1 (4\%) patient of those having PATE without arterial hypotension; total mortality was $18 \%$. There was progressive deterioration in $32 \%$ of the patients due to the progression of both respiratory and cardiovascular failures correctable in the prehospital stage in more than 32 and $39 \%$ of cases, respectively.

Адрес для корреспонденции (Correspondence to): Key words: pulmonary artery thromboembolism, prehospital

Ермолаев Андрей Александрович

E-mail: ermolaev5000@rambler.ru stage, mortality. 
В широкой дискуссии, посвященной вопросам диагностики и лечения тромбоэмболии легочной артерии (ТЭЛА), направление эффективного ведения больных с ТЭЛА без артериальной гипотонии при первичном обращении занимает особое место. Результатами многочисленных исследований последних 10 лет показано, что своевременность верификации диагноза именно у данного контингента больных во многом определяет перспективы снижения летальности при ТЭЛА в целом [1-6]. Однако столь же хорошо охарактеризованы и ограничения своевременной диагностики, обусловленные отсутствием специфичности клинических проявлений заболевания в острейшем периоде [6-10]. Очевидно, что последнее приобретает особую актуальность в условиях догоспитального этапа.

Цель исследования - оценить динамику состояния функций дыхания и кровообращения в острейшем периоде у больных с ТЭЛА без артериальной гипотонии при первичном обращении.

Задачи исследования:

1. Оценка общей и догоспитальной летальности у больных с ТЭЛА без гипотонии при первичном обращении.

2. Анализ динамики проявлений дыхательной и сердечной недостаточности у данной категории больных в течение догоспитального периода.

3. Сравнение ЭКГ-изменений в острейшем периоде ТЭЛА до и после начала терапии на догоспитальном этапе.

\section{Материал и методы}

Проведен анализ 28-и случаев ТЭЛА без снижения артериального давления при первичном осмотре на догоспитальном этапе: у 27-и больных первичный диагноз догоспитального этапа был подтвержден в условиях стационара; в 1 случае у умершего на догоспитальном этапе больного - при патоморфологическом исследовании.

Анализ выполнен по данным медицинской документации Станции скорой и неотложной медицинской помощи Департамента здравоохранения г. Москвы, отделения скорой медицинской помощи ФГУ «Поликлиника № 1» УД Президента РФ, НИИ скорой помощи им. Н.В. Склифосовского Департамента здравоохранения г. Москвы, Центральной клинической больницы МЦ УдП РФ, судебно-медицинского морга № 5 (при ГКБ № 67 Департамента здравоохранения г. Москвы) (табл. 1)

Критерием включения больных в исследование являлся уровень САД при первичном осмотре на догоспитальном этапе не менее 100 мм рт. ст.

В исследование вошло 17 мужчин и 11 женщин, средний возраст больных составил $67,5 \pm 14,5$ лет.
Продолжительность догоспитального этапа составила $87,0 \pm 29,7$ мин. Терапия ТЭЛА осуществлялась бригадами СМП в соответствии со Стандартами оказания экстренной медицинской помощи на догоспитальном этапе в г. Москва и включала следующие направления: оксигенотерапия, антикоагулянты (гепарин, клексан), дезагреганты, анальгетики (в том числе наркотические), инфузионная терапия, симптоматическая терапия [11].

Выполнено сравнение клинической и ЭКГ-картины при первичном осмотре и в динамике на догоспитальном этапе, а также при поступлении больного в стационар. Статистическая обработка производилась с использованием программы «Statistica 6.0». Достоверность различий оценивалась при помощи точного одностороннего критерия Фишера и рангового критерия Манна-Уитни.

\section{Результаты и обсуждение}

Летальный исход на догоспитальном этапе зафиксирован в 1 случае (4\%), впоследствии в стационаре скончались еще 4 больных (в среднем через 8,0 6,5 суток от поступления). Таким образом, общая летальность у больных с ТЭЛА без артериальной гипотонии при первичном обращении составила 18\% (5 из 28).

Диагноз ТЭЛА на догоспитальном этапе основывался на жалобах, анамнестических сведениях, результатах физикального осмотра, динамике данных ЭКГ.

Наиболее характерной жалобой являлось ощущение затруднения дыхания (удушье, чувство нехватки воздуха), которое отмечали 96\% больных (27 из 28). В 39\% случаях (11 больных) отмечался кашель. В 7-и случаях (25\%) больными была отмечена боль в грудной клетке, при этом только в 3-х случаях боль носила ангинозный характер. Помимо изложенного, больные свидетельствовали о наличии слабости $-25 \%$ (7 случаев), сердцебиения - 21\% (6 случаев), боли в одной ноге $18 \%$ (5 случаев), эпизодов кратковременной потери сознания - 11\% (3 случая), кровохарканья - 4\% (1 случай), боли в животе - 4\% (1 случай).

В $86 \%$ случаев (24 из 28) у больных в анамнезе имели место различные факторы риска, являющиеся, по данным литературы, предрасполагающими к возникновению ТЭЛА $[12,13]$. Чаще всего отмечались пожилой возраст (старше 65 лет) - 57\% (16 больных), варикозная болезнь вен нижних конечностей и/или хроническая венозная недостаточность - 36\% (10 больных), хроническая легочно-сердечная недостаточность - 29\% (8 больных), перенесенные ранее ТЭЛА и/или тромбоз глубоких вен нижних конечностей $-21 \%$ (6 больных). Реже регистрировались прочие факторы риска развития

Источники медицинской документации

Таблица 1

\begin{tabular}{lc}
\hline Догоспитальный этап & $n$ \\
\hline Станция скорой и неотложной медицинской помощи & 22 \\
им. А. С. Пучкова Департамента здравоохранения г. Москвы & 6 \\
Отделение скорой медицинской помощи ФГУ «Поликлиника № 1» УдП РФ & $\boldsymbol{n}$ \\
Стационар/морг & 21 \\
НИИ СП им. Н. В. Склифосовского Департамента здравоохранения г. Москвы & 6 \\
Центральная клиническая больница МЦ УДП РФ & 1 \\
Судебно-медицинский морг № 5 (при ГКБ № 67 Департамента здравооранения г. Москвы) & $\mathbf{2 8}$ \\
Всего & \\
\hline
\end{tabular}


ТЭЛА: перенесенный инсульт со стойким неврологическим дефицитом в виде гемипареза или гемиплегии 14\% (4 больных), ожирение - 11\% (3 больных), длительный постельный режим - 7\% (2 больных), злокачественное новообразование - 7\% (2 больных), недавнее (до 1 месяца) протезирование тазобедренного сустава 4\% (1 больной), заместительная гормональная терапия - 4\% (1 больной).

При физикальном первичном осмотре на догоспитальном этапе наиболее часто выявлялись следующие характерные для ТЭЛА симптомы: тахипноэ (ЧДД>20 в мин) $-75 \%$ (21 случай), тахикардия (ЧСС $\geqslant 100$ в мин) $-57 \%$ (16 случаев), цианоз - 39\% (11 больных), локальное ослабление дыхания и хрипы в легких - по 36\% (10 случаев), клинические признаки тромбоза глубоких вен нижних конечностей - 18\% (5 случаев), акцент II тона на легочной артерии - 11\% (3 случая). При аускультации выявлялись влажные хрипы в легких у 7-и больных (25\%), сухие хрипы у 5 -и больных (18\%). Следует отметить, что у 2-х больных имелось сочетание влажных и сухих хрипов. Также отмечались бледность кожных покровов - 29\% (8 больных), потливость и гипертермия - в 21\% случаев (6 больных), периферические отеки - 18\% (5 больных), гепатомегалия и локальное притупление при перкуссии грудной клетки - в 11\% (3 больных).

При ЭКГ-исследовании, которое было выполнено на догоспитальном этапе у 26-и больных (93\%), характерные для ТЭЛА признаки перегрузки правых отделов сердца (отклонение ЭОС вправо, блокада правой ножки пучка Гиса, P-pulmonale, SI-QIII, отрицательные зубцы $\mathrm{T}$ в отведениях V1-V3, глубокие зубцы $\mathrm{S}$ в отведениях $\mathrm{V} 5-\mathrm{V} 6$ ) регистрировались в $73 \%$ случаев (19 из 26).

С учетом вышеизложенного, диагноз ТЭЛА на догоспитальном этапе был в большинстве случаев основан на наличии симптомов острой дыхательной недостаточности (тахипноэ, цианоз) нередко в сочетании с нарушениями со стороны сердечно-сосудистой системы (тахикардия, ЭКГ-изменения) у больных с жалобами на удушье (чувство нехватки воздуха) и анамнестическими факторами риска возникновения венозных тромбозов.

Анализ течения острейшего периода ТЭЛА в динамике ее клинических проявлений выполнен нами по следующим направлениям, отражающим тяжесть состояния больных: характер изменения уровня сознания, наличие и прогрессирование острой дыхательной и сердечно-сосудистой недостаточностей.

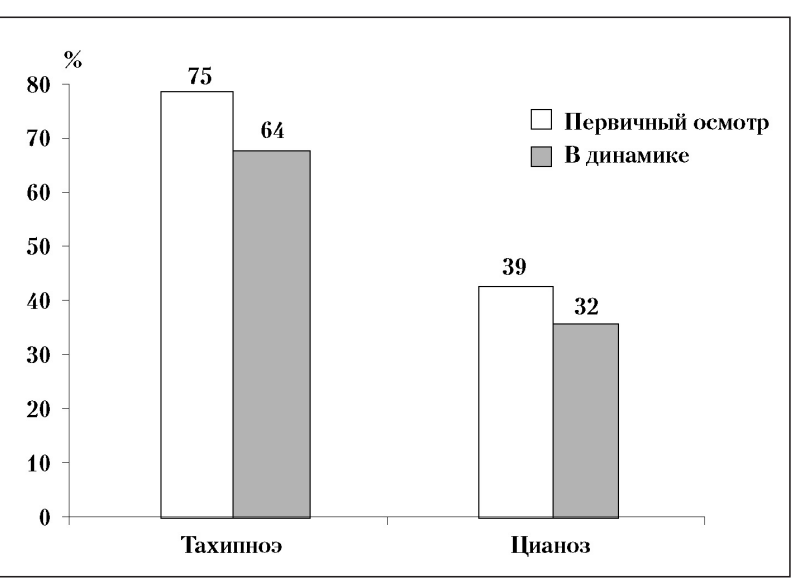

Рис. 1. Динамика симптомов дыхательной недостаточности у больных с ТЭЛА без артериальной гипотонии при первичном обращении на фоне терапии на догоспитальном этапе $(n=28)$.

При первичном осмотре у 27-и больных из 28-и (96\%) сознание было расценено как ясное, в 1-м случае (4\%) - как оглушение. При повторном (в среднем через 87,0 29,7 мин) осмотре ясное сознание сохранялось у 26-и (93\%) больных, в 1-м случае сознание было угнетено до уровня оглушения, в 1-м случае - до уровня комы (3 балла по ШКГ). Таким образом, у большинства больных с ТЭЛА без артериальной гипотонии при первичном обращении сознание было расценено сотрудниками СМП как ясное.

Проявления дыхательной недостаточности в динамике оценивались на основании изменений ЧДд, степени выраженности цианоза (рис. 1). На фоне проведенной бригадами СМП терапии частота встречаемости тахипноэ уменьшилась с 75\% (21 из 28 случаев) при первичном осмотре до 64\% (18 из 28) в динамике $(p=0,07)$. Средняя ЧДД уменьшилась с $24,0 \pm 5,7$ в мин до $21,0 \pm 5,6$ в мин, соответственно ( $p=0,02)$. Проявления цианоза также имели тенденцию к уменьшению - с 39\% (11 больных) до 32\% (9 больных) $(p=0,4)$.

Уменьшение степени выраженности симптомов дыхательной недостаточности было зарегистрировано на фоне терапии у 9-и (32\%) больных из 28. В данной подгруппе средняя ЧДД уменьшилась с $28,0 \pm 6,5$ до $20,0 \pm 1,8$ в мин. Частота тахипноэ уменьшилась с 89\% (у 8-и больных из 9-и) до 33\% (у 3-х больных из 9-и). Цианоз, наблюдавшийся первично в $67 \%$ случаев (6 больных), после проведенной на догоспитальном этапе терапии регистрировался уже в $22 \%$ случаев (2-е больных).

Таблица 2

Динамика симптомов дыхательной недостаточности у больных с ТЭЛА без артериальной гипотонии при первичном обращении в зависимости от ответа на терапию на догоспитальном этапе

\begin{tabular}{|c|c|c|c|c|c|c|}
\hline \multirow[t]{3}{*}{ Симптомы } & \multicolumn{6}{|c|}{ Значения показателей в подгруппах на этапах исследования } \\
\hline & \multicolumn{3}{|c|}{ с эффектом от терапии, $n=9$ (100\%) } & \multicolumn{3}{|c|}{ без эффекта от терапии, $n=19$ (100\%) } \\
\hline & до терапии & после терапии & $p$ & до терапии & после терапии & $p$ \\
\hline Наличие ОДН & $9(100 \%)$ & $5(56 \%)$ & 0,04 & $14(74 \%)$ & $18(95 \%)$ & 0,09 \\
\hline ЧДД в мин & $28,0 \pm 6,5$ & $20,0 \pm 1,8$ & 0,003 & $24,0 \pm 4,4$ & $22,0 \pm 6,7$ & 0,6 \\
\hline Тахипноэ & $8(89 \%)$ & $3(33 \%)$ & 0,03 & $13(68 \%)$ & $15(79 \%)$ & 0,4 \\
\hline Цианоз & $6(67 \%)$ & $2(22 \%)$ & 0,08 & $5(26 \%)$ & $7(37 \%)$ & 0,4 \\
\hline
\end{tabular}

Примечание. ОДН - острая дыхательная недостаточность; ЧдД - частота дыхательных движений. 
Таблица 3

Динамика ЭКГ-изменений у больных с ТЭЛА без артериальной гипотонии при первичном обращении в зависимости от наличия тахикардии

\begin{tabular}{|c|c|c|c|c|c|c|}
\hline \multirow[t]{2}{*}{ ЭКГ-изменения } & \multicolumn{3}{|c|}{$\begin{array}{l}\text { В подгруппе больных } \\
\text { с тахикардией }(n=15)\end{array}$} & \multicolumn{3}{|c|}{$\begin{array}{c}\text { В подгруппе больных } \\
\text { без тахикардии }(n=10)\end{array}$} \\
\hline & первично & в динамике & $p$ & первично & в динамике & $p$ \\
\hline $\mathrm{HPC}^{1}$ & $15(100 \%)$ & $15(100 \%)$ & - & $2(20 \%)$ & $4(40 \%)$ & 0,3 \\
\hline Перегрузка правых отделов & $13(87 \%)$ & $11(73 \%)$ & 0,3 & $6(60 \%)$ & $6(60 \%)$ & 0,7 \\
\hline Изменения ST & $3(20 \%)$ & $2(13 \%)$ & 0,5 & $1(10 \%)$ & $1(10 \%)$ & 0,8 \\
\hline
\end{tabular}

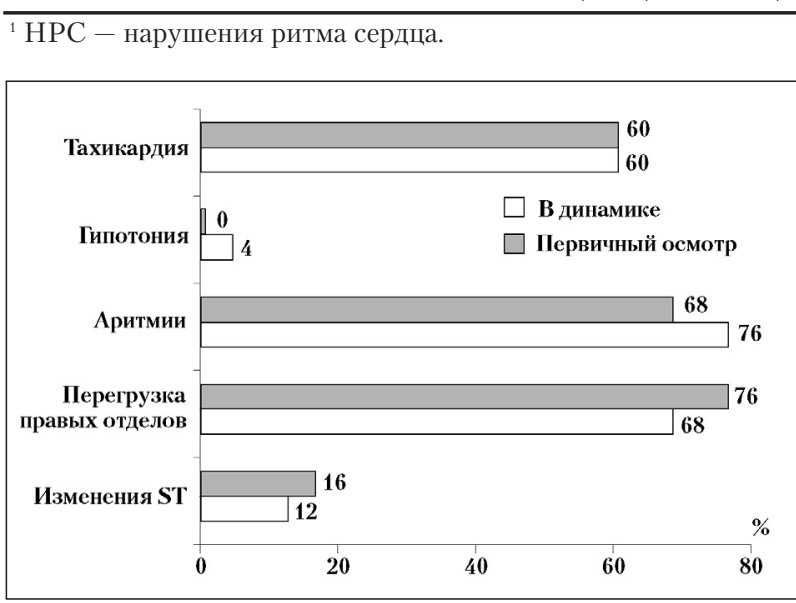

Рис. 2. Динамика симптомов недостаточности кровообращения в острейшем периоде ТЭЛА у больных без артериальной гипотонии при первичном обращении $(n=25)$.

В 19-и случаях (68\%) проводимая терапия не привела к уменьшению степени выраженности симптомов дыхательной недостаточности. Несмотря на небольшое снижение средней ЧДД в динамике в данной подгруппе

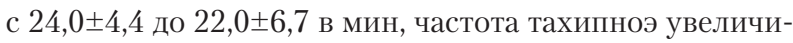
лась с 68\% (13 больных) до 79\% (15 больных), а цианоз с 26\% (5 больных) до 37\% (7 больных).

Оценка динамики степени выраженности симптомов сердечно-сосудистой недостаточности производилась в сочетании с анализом данных ЭКГ и была выполнена у 25-и больных.

Установлено, что средние ЧСС, САД и ДАД составили при первичном осмотре $100,0 \pm 19,1$ в мин, 140,0 $\pm 21,9$ мм рт. ст. и 80,0土11,5 мм рт. ст., соответственно; после проведенной на догоспитальном этапе терапии (то есть, в среднем, через $87,0 \pm 29,7$ мин) - 100,0 028,2 в мин, $130,0 \pm 36,8$ мм рт. ст. и $80,0 \pm 21,9$ мм рт. ст., соответственно $(p>0,5)$. Следует отметить, что в динамике у 1 больного $(4 \%)$ развилась гипотония. Тахикардия (ЧСС $\geqslant 100$ в мин) наблюдалась у 60\% (15 из 25) больных, как при первичном осмотре, так и в динамике. Тахикардия у больных с ТЭЛА во всех случаях сочеталась с ЭКГ-изменениями.

У больных с тахикардией, как при первичном осмотре, так и в динамике на ЭКГ в 100\% случаев (15 из 15) регистрировали нарушения ритма (преимущественно синусовая тахикардия). На фоне проведенной на догоспитальном этапе терапии у больных с тахикардией отмечалось уменьшение признаков перегрузки правых отделов сердца на ЭКГ, которые наблюдались первично в 87\% случаев (13 из 15), в динамике - в 73\% (11 из 15), и изменений сегмента ST - 20\% случаев (3 из 15) и 13\% (2 из 15), соответственно.

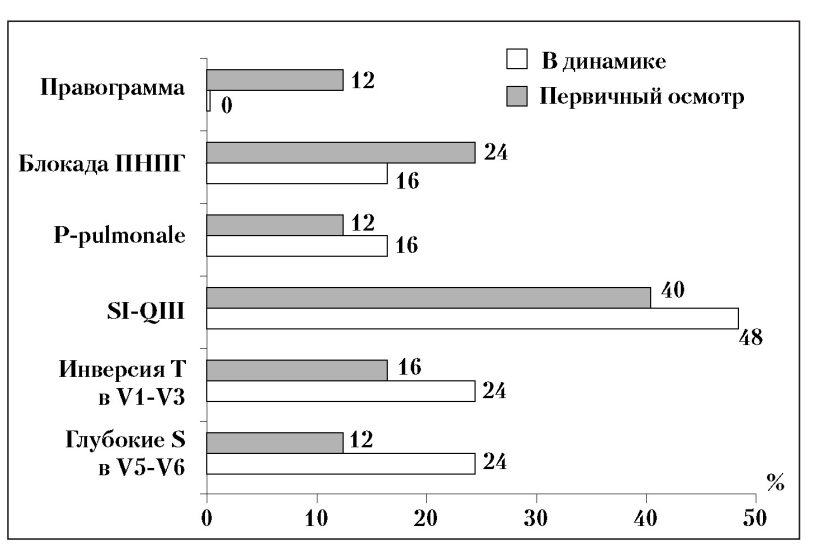

Рис. 3. Динамика ЭКГ-признаков перегрузки правых отделов сердца в острейшем периоде ТЭЛА у больных без артериальной гипотонии при первичном обращении $(n=25)$.

У больных без тахикардии $(n=10)$ первично на ЭКГ нарушения ритма регистрировали в $20 \%$ случаев ( 2 из 10), в динамике в 2 раза чаще $-40 \%$ ( 4 из 10). Признаки перегрузки правых отделов сердца и изменения сегмента ST на ЭКГ у больных без тахикардии отмечались с одинаковой частотой как до, так и после начала терапии на догоспитальном этапе - $60 \%$ (6 из 10) и $10 \%$ (1 из 10), соответственно (табл. 3).

Среди 25-и больных с ТЭЛА нарушения сердечного ритма, признаки перегрузки правых отделов сердца и изменения сегмента ST на ЭКГ имели место первично в $17-и$ (68\%), 19-и (76\%) и 4-х (16\%) случаях, в динамике - в 19-и (76\%), 17-и (68\%) и 3-х (12\%) случаях, соответственно ( $p>0,3)$ (рис. 2).

Среди нарушений ритма чаще всего наблюдалась синусовая тахикардия - 11 (44\%) первично и 12 (48\%) случаев в динамике $(p=0,5)$. С одинаковой частотой при первичной и повторной регистрации ЭКГ выявлялись фибрилляция/трепетание предсердий - 4 (16\%) больных, наджелудочковая и желудочковая экстрасистолия - по 1 (4\%) случаю. Миграция водителя ритма зарегистрирована у 1 больного на первичной ЭКГ, в динамике однократно определялись синусовая аритмия и идиовентрикулярный ритм.

Распространенность и динамика признаков перегрузки правых отделов представлена на рис. 3. Отклонение ЭОС вправо первично наблюдалось у 3-х больных $(12 \%)$ и не было зафиксировано в динамике ни в одном из случаев $(p=0,1)$. Блокада правой ножки пучка Гиса также чаще наблюдалась при первичном ЭКГ-исследовании -6 случаев (24\%), чем в динамике - 4 слу- 
чая $(16 \%)(p=0,4)$. Отмечено нарастание в динамике $(p>0,2)$ таких признаков перегрузки правых отделов сердца как P-pulmonale - 3 (12\%) и 4 (16\%) случаев, соответственно $(p=0,5)$, SI-QIII - $10(40 \%)$ и $12(48 \%)$ $(p=0,39)$, инверсия зубцов Т в отведениях V1-V3-4 $(16 \%)$ и $6(24 \%)(p=0,36)$, наличие глубоких зубцов $\mathrm{S}$ в отведениях V5-V6 - 3 (12\%) и $6(24 \%)(p=0,23)$.

При первичной регистрации ЭКГ наблюдались следующие изменения сегмента ST: элевация ST в нижних отведениях (II, III, AVF) - 1 больной (4\%); элевация ST в нижних отведениях в сочетании с депрессией ST в передних отведениях (I, AVL, V1-V6) - 1 больной (4\%); изолированная депрессия ST в передних отведениях - 1 больной (4\%); депрессия ST в передних и нижних отведениях - 1 больной (4\%). Изменения ST, указанные в 2-х последних случаях, сохранились в динамике, у 1-го больного появился подъем ST в передних отведениях. У 2-х больных (8\%) с подъемом ST в отведениях II, III, AVF при первичном ЭКГ-исследовании каких-либо изменений со стороны указанного сегмента в динамике не наблюдалось.

Исходно нарушения ритма сочетались с признаками перегрузки правых отделов у 15-и больных (60\%), с изменениями ST - у 4-х больных (16\%). В динамике указанные сочетания наблюдались в 12 -и (48\%) и 3 -х (12\%) случаях, соответственно. Одновременно нарушения ритма, признаки перегрузки правых отделов и изменения ST имели место у 4-х больных (16\%) первично и у 1 -го больного (4\%) в динамике. Изолированного сочетания признаков перегрузки правых отделов и изменений ST без нарушений ритма не было зафиксировано ни в одном из случаев (до и после начала терапии на догоспитальном этапе). Ни одного из указанных изменений не было выявлено при первичной регистрации ЭКГ у 4-х больных (16\%), в динамике - у 1-го больного (4\%).

Следует отметить, что при первичном осмотре у 16\% больных (4 из 25) не было выявлено объективных признаков, свидетельствующих об острой недостаточности кровообращения. В динамике, несмотря на проводимую на догоспитальном этапе терапию, объективных признаков острой недостаточности кровообращения не было выявлено только у 1-го больного (4\%).

В целом, на фоне терапии, проведенной на догоспитальном этапе, явления недостаточности кровообращения уменьшились у 7 -и больных (39\%). В указанной подгруппе средняя ЧСС снизилась со 100,0 $\pm 24,6$ до 90,0 $\pm 20,6$ в мин. Число больных с тахикардией уменьшилось с 57\% (4 из 7) до 43\% (3 из 7). Признаки перегрузки правых отделов сердца регистрировались в динамике значительно реже $-29 \%$ (2 из 7), чем до терапии $-71 \%$ (5 из 7).

Среди больных, у которых в динамике не отмечено уменьшения явлений недостаточности кровообращения $(n=18)$, средняя ЧСС увеличилась в динамике со $100,0 \pm 16,2$ до $102,5 \pm 31,1$ в мин. Тахикардия наблюдалась на $6 \%$ чаще в динамике, чем при первичном осмотpe $-61 \%$ (11 больных) и 67\% (12 больных), соответственно. Также отмечалось увеличение числа больных с ЭКГ-признаками перегрузки правых отделов сердца с 78\% (14 больных) до 83\% (15 больных).
Таким образом, у больных с ТЭЛА без артериальной гипотонии при первичном обращении в $84 \%$ случаев исходно имеются объективные признаки сердечнососудистой недостаточности, которые, несмотря на проводимую терапию, через 87,0 29,7 мин были зафиксированы уже более чем у $95 \%$ больных.

Показатели гемодинамики у данной категории больных относительно устойчивы в течение догоспитального этапа (ЧСС в среднем от 100,0 19,1 до $100,0 \pm 28,2$ в мин, САД и ДАД от $140,0 \pm 21,9$ мм рт. ст. и $80,0 \pm 11,5$ мм рт. ст. до $130,0 \pm 36,8$ мм рт. ст. и $80,0 \pm 21,9$ мм рт. ст., соответственно). Тахикардия в $73-100 \%$ случаев сочетается с нарушениями ритма и признаками перегрузки правых отделов сердца на ЭКГ. Характерно нарастание в динамике некоторых симптомов перегрузки правых отделов сердца (SI-QIII, P-pulmonale, инверсия T в отведениях $\mathrm{V} 1-\mathrm{V} 3$, появление глубоких $\mathrm{S}$ в отведениях V5-V6). Изменения сегмента ST на ЭКГ в острейшем периоде ТЭЛА у больных с ТЭЛА без артериальной гипотонии при первичном обращении носят нестойкий характер и наблюдались в $16 \%$ случаев исходно и в $12 \%$ случаев при повторной регистрации ЭКГ.

При первичном осмотре состояние 11-и из 28-и больных с ТЭЛА (39\%) расценено как тяжелое, в 16-и случаях (57\%) - средней тяжести, в 1-м случае (4\%) - удовлетворительное. При осмотре в динамике состояние 3 -х больных из 28-и (11\%) расценено как удовлетворительное, 11-и больных (39\%) - средней тяжести, 12-и больных (43\%) тяжелое и 2-х больных (7\%) - крайне тяжелое (рис. 4).

В целом, увеличение тяжести состояния больных отмечалось в $32 \%$ случаев (9 из 28). Отрицательная динамика в 1-м случае была обусловлена усугублением явлений дыхательной недостаточности, в 3-х случаях - сердечной недостаточности и в 3-х случаях - сочетанным нарастанием признаков нарушения функций дыхания и кровообращения. Крайняя тяжесть состояния 2-х больных объяснялась агональным состоянием больного на момент повторного осмотра в одном случае и угнетением сознания (до 10-11 баллов по ШКГ) во втором случае.

По нашему мнению, далее представленный клинический пример в полной мере отражает возможные

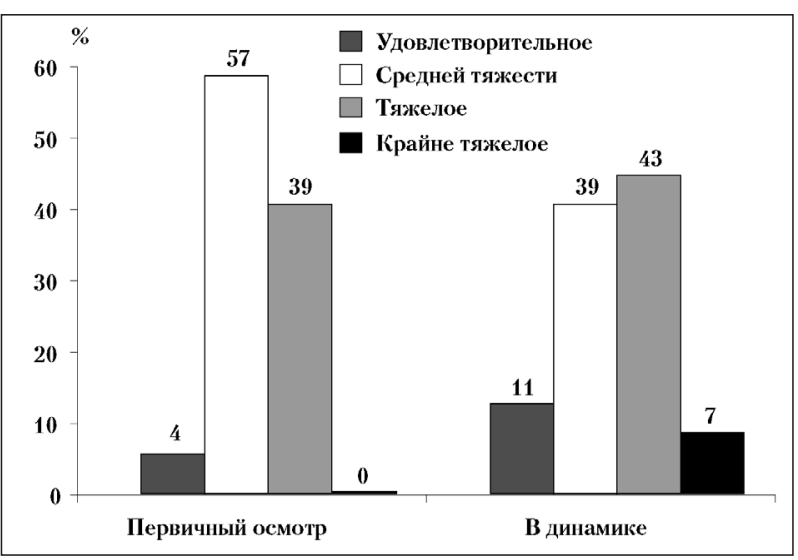

Рис. 4. Динамика тяжести состояния больных с ТЭЛА без артериальной гипотонии при первичном обращении $(n=28)$. 
темпы прогрессирования клинических проявлений тромбоэмболии легочной артерии. Считаем необходимым особо отметить отсутствие гипотонии у больного при его первичном осмотре сотрудниками СМП.

Больной Ж., 58 лет. Повод к вызову СМП: впервые возникшее удушье.

По прибытии линейной фельдшерской бригады СМП в 12 ч 36 мин (через 9 минут от момента поступления вызова по «03») больной предъявлял жалобы на нехватку воздуха, удушье, интенсивную боль за грудиной, чувство страха.

Из анамнеза: ухудшение состояния возникло около 20 минут назад. Накануне (со слов больного «приблизительно неделю назад») перенес простудное заболевание, самостоятельно принимал ампициллин, анальгин, таблетки от кашля (к врачу не обращался). Хронические заболевания: варикозная болезнь вен нижних конечностей. Аллергоанамнез не отягощен.

Объективно (12 ч 36 мин): Состояние тяжелое. Сознание ясное. Неврологический статус: без особенностей. Ортопноэ. Кожные покровы влажные, серого цвета, отеков нет, $\mathrm{t}-35,7^{\circ} \mathrm{C}$. Дыхание затруднено, ЧДД - 32 в мин, аускультативно везикулярное, хрипов нет. Пульс ритмичный, удовлетворительного наполнения. $\mathrm{Ps}=\mathrm{UCC}=116$ в мин. Тоны сердца приглушены, шумов нет. АД - 130/90 мм рт. ст. (рабочее 130/80 мм рт. ст.) Живот мягкий, безболезненный. Печень у края реберной дуги, селезенка не увеличена. Физиологические отправления не нарушены. Мочеполовая система без особенностей.

ЭКГ: синусовая тахикардия, ЧСС - 116 в мин. Вертикальная ЭОС. Блокада правой ножки пучка Гиса. SI-QIII. Подъем ST в III отведении до 2 мм. Отрицательные зубцы T в отведениях III, AVF, V1-V4. Глубокие зубцы S в отведениях V5-V6.

Первичное заключение СМП: ТЭЛА.

В 12 ч 58 мин вызвана специализированная бригада. До прибытия спецбригады проведена терапия: катетеризация кубитальной вены, оксигенотерапия, гепарин 2000 ед в/в, аспирин 250 мг сублингвально, морфин 10 мг в/в. Несмотря на проведенную терапию состояние больного с ухудшением: одышка усилилась, кожные покровы цианотичные, покрыты холодным липким потом, АД не определяется. В 13 ч 05 мин больной потерял сознание, зафиксирована остановка сердечной деятельности и дыхания. Начаты реанимационные мероприятия: непрямой массаж сердца, ИВЛ.

На момент прибытия специализированной бригады СМП (13 ч 10 мин) больной в состоянии клинической смерти, проводятся реанимационные мероприятия. Сознание отсутствует. Кожные покровы влажные, цианотичные. Самостоятельного дыхания нет. Пульс, АД не определяются. Зрачки широкие, $\mathrm{OD}=\mathrm{OS}$, фотореакция вялая. На ЭКГ идиовентрикулярный

\section{Литература}

1. Stein P., Beemath A., Matta F. et al. Enlarged right ventricle without shock in acute pulmonary embolism: prognosis. Am. J. Med. 2008; 121 (1): 34-42.

2. Becattini C., Agnelli G., Salvi A. et al. Bolus tenecteplase for right ventricle dysfunction in hemodynamically stable patients with pulmonary embolism. Thromb. Res. 2009; 125 (3): e82-e86.

3. Wood K. E. A history of pulmonary embolism and deep venous thrombosis. Crit. Care Clin. 2009; 25 (1): 115-131.

4. Неймарк М. И., Зальиман А. Г., Акатов А. В. и соавт. Пути улучшения результатов лечения тромбоэмболии лёгочной артерии. Общая реаниматология 2010; VI (2): 48-52.

5. Ozsu S., Karaman K., Mentese A. et al. Combined risk stratification with computerized tomography/echocardiography and biomarkers in patients with normotensive pulmonary embolism. Thromb. Res. 2010; 126 (6): 486-492.

6. Ceylan N., Tasbakan S., Bayraktaroglu S. et al. Predictors of clinical outcome in acute pulmonary embolism: correlation of CT pulmonary angiography with clinical, echocardiography and laboratory findings. Acad. Radiol. 2011; 18 (1): 47-53.

7. Никифоров Ю. В., Чернышёва Г. Г., Вуколова О. В. и соавт. Роль Dдимеров плазмы крови в диагностике тромботических состояний у ритм, ЧСС - 29 в мин. Продолжены реанимационные мероприятия: интубация трахеи, ИВЛ мешком Амбу, непрямой массаж сердца, адреналин 1 мг в/в каждые 5 мин, атропин 3 мг в/в. В течение 30 мин сердечно-легочная реанимация проводилась в полном объеме - без эффекта. На ЭКГ асистолия. В 13 ч 40 мин констатирована биологическая смерть.

Диагноз, установленный врачами СМП: тромбоэмболия легочной артерии. Клиническая смерть, состояние после неэффективных реанимационных мероприятий. Смерть при бригаде СМП.

При патологоморфологическом исследовании (5-й судебно-медицинский морг) диагноз тромбоэмболии легочной артерии подтвержден.

\section{Выводы}

1. Летальность больных с ТЭЛА без артериальной гипотонии при первичном обращении составила $18 \%$, летальность на догоспитальном этапе $-4 \%$. Несмотря на проводимую терапию, установлено прогрессивное ухудшение тяжести состояния $32 \%$ больных за счет нарастания явлений как дыхательной, так и сердечно-сосудистой недостаточности.

2. Для большинства больных с ТЭЛА без артериальной гипотонии при первичном обращении (96\%) характерно отсутствие нарушения сознания.

3. У $82 \%$ больных с ТЭЛА без артериальной гипотонии при первичном обращении имеются проявления дыхательной недостаточности, которые поддаются коррекции на догоспитальном этапе не более чем в $32 \%$ случаев.

4. Несмотря на отсутствие снижения артериального давления, физикальные и ЭКГ-признаки, свидетельствующие об острой недостаточности кровообращения, первично установлены у 84\% больных с их последующим прогрессированием у 96\% больных в течение догоспитального периода. Некоторое уменьшение симптомов недостаточности кровообращения на фоне проводимой на догоспитальном этапе терапии наблюдается не более чем в 39\% случаев.

5. На ЭКГ у больных с ТЭЛА без артериальной гипотонии при первичном обращении характерно нарастание в динамике таких признаков перегрузки правых отделов сердца, как P-pulmonale, SI-QIII, инверсия зубцов Т в отведениях V1-V3, глубокие зубцы S в отведениях V5-V6.

больных с хронической ишемической болезнью сердца. Общая реаниматология 2006; II (5-6): 66-69.

8. Douma R. A., Kessels J. B., Buller H. R., Gerdes V. E. Knowledge of the D-dimer test result influences clinical probability assessment of pulmonary embolism. Thromb. Res. 2010; 126 (4): e271-e275.

9. Vanni S., Polidori G., Vergara R. et al. Prognostic value of ECG among patients with acute pulmonary embolism and normal blood pressure. Am. J. Med. 2009; 122 (3): 257-264.

10. Hartmann I., Wittenberg R., Schaefer-Prokop C. Imaging of acute pulmonary embolism using multi-detector CT angiography: An update on imaging technique and interpretation. Eur. J. Radiol. 2010; 74 (1): 40-49.

11. Стандарты оказания медицинской помощи больным и пострадавшим бригадами Станции скорой и неотложной медицинской помощи им. А. С. Пучкова. М.; 2006.

12. Anderson F. A. Jr., Spencer F. A. Risk factors for venous thromboembolism. Circulation 2003; 107 (23 Suppl 1): I9-I16.

13. Torbicki A., Perrier A., Konstantinides S. et al. Guidelines on the diagnosis and management of acute pulmonary embolism: the Task Force for the Diagnosis and Management of Acute Pulmonary Embolism of the European Society of Cardiology (ESC). Eur. Heart J. 2008; 29 (18): 2276-2315.

Поступила 22.04.11 